\title{
Interactive comment on "Retrieval of rainfall fields in urban areas using attenuation measurements from mobile phone networks: a modeling feasibility study" by Bahtiyor Zohidov et al.
}

\section{Bahtiyor Zohidov et al.}

bahtiyor.zohidov@external.eumesat.int

Received and published: 12 January 2017

We really thank the useful and constructive comments and suggestions given by Reviewers. These suggestions will be very helpful to improve the manuscript, and they will fully be taken in account. This note combines our responses to the general comments and to the main specific comments raised by both Reviewers. This research subject, measurement of rainfall from attenuation data along microwave links (ML), has been launched and developed by a group of pioneering scientists. We have started more recently our work on that subject with an approach mixing urban hydrology and modelling of urban systems. We follow the idea that ML networks have a great potential for the

Printer-friendly version

Discussion paper 
hydrometeorological survey of urban areas (Messer et al., 2012). An interdisciplinary collaboration could help its development for applications in cities and facilitate access to commercial ML data.

\section{Response to Reviewer \#1}

1. We do not refer to the paper by Vignal et al. (2003) because it is not related to the work presented in this manuscript. The objective of Vignal et al. (2003) was to correct weather radar data from attenuation by rainfall and to improve the existing methods (for instance Hitschfeld-Bordan), known to be numerically unstable. In the present manuscript, weather radar images are only used as realistic rainfall fields to give us a ground truth. We use them to initiate our simulation and do quality reconstruction measurements. We first simulate the attenuation measurements that could be obtained from the networks of ML built by mobile telephone companies. Then the presented method proposes to reconstruct rainfall fields from these attenuation measurements. The common point of these two studies is the use of the same inverse algorithm, initially developed by Tarentola and Valette (1982), and reported in a very nice way in the text book by Menke (1989). This algorithm has become very classical method to solve linear and non-linear problems in Geoscience. A very interesting point of this method is the importance attached to the a priori information which may have a decisive influence for non-linear or/and underdetermined problems. This particular aspect is very helpful to define the applications conditions (initialization or a priori information) adapted to the problem to solve. If the paper by Vignal et al. (2003) and this manuscript use this algorithm, the application conditions are different and very specific to each study. Concerning this manuscript, we focused on defining the a priori information that is able to adapt to a quantity of data (density of $\mathrm{ML}$ ) which decreases from the city center to the suburb. The presented nested initialization refining the spatial resolution of the rainfall field, according to the density of $\mathrm{ML}$, allows to satisfy this constraint.

2. We agree that the expression "feasibility study" may lack of clarity and deserves to be better explained. A key issue on this subject is the difficulty to collect actual obser-

Printer-friendly version

Discussion paper 
vation data. For instance, the used network in Nantes regroups ML from four mobile phone companies. Organizing an experiment with actual data is very time consuming regarding the fact that collecting this information from all these companies is not an easy task. A first step in this study was to simulate realistic attenuation data to develop and assess the reconstruction method. This first step made us check if our reconstruction method deserves an evaluation in a real-world context. The "feasibility study" denotes this first step. It is close to the approach followed by Cuccoli et al. (2011).

The Reviewer suggests that we better differentiate our contribution from other studies. We agree that this part of the introduction could be improved, and we confirm that this differentiation will be added to the revised version of the manuscript. This very active research subject has mainly been addressed by pioneering researchers that are cited in the manuscript who pursue complementary objectives: i) measurement of rainfall by considering $\mathrm{ML}$ as rainfall measurement devices, and mapping of rainfall fields over large areas by using interpolation methods, ii) use of $\mathrm{ML}$ to adjust radar data, or to complement raingauges networks, iii) reconstruction of rainfall fields in urban areas using a dense network of ML, iv) a mixed approach proposed by Zinevich et al. (2008) which reconstructs rainfall fields from a network of $M L$ displaying a very heterogeneous spatial density and which combines retrieval and interpolation. The contribution of our manuscript submitted to HESS aims to progress toward the mapping of rainfall fields in urban areas not surveyed by classical devices (i.e. raingauge network, weather radar). At this geographic scale, path-integrated attenuation provided by $\mathrm{ML}$ cannot be considered as quasi-punctual, which prevents using classical interpolation methods. This manuscript complements the study by Cuccoli et al. (2013). It differs from the contribution of these authors by several points: i) the retrieval method ; Cuccoli et al. (2003) based their reconstruction on a tomographic approach while we use explicitly the inverse algorithm taking into account the a priori information for this manuscript, ii) the capability of our method to adapt to more irregular link networks thanks to this specificity, as illustrated by the case study in the manuscript while the case study presented by our colleagues uses a spatially homogeneous and regular network. The

Printer-friendly version

Discussion paper
Interactive comment 
review raises the comparison with the existing algorithms. To the best of our knowledge, such a comparison has not yet been done. We support this suggestion which could be the subject of a future collaborative research project. Nevertheless, this comparison needs to be carefully prepared and for instance the data used for that purpose: numerical experiments or real-world data is not a negligible aspect.

3. In this general comment which summarizes specific comments, the Reviewer raises the realism of the numerical experiments which serve to conduct the feasibility study. We clearly understand from the Reviewer comments and suggestions, that the assumptions on which the study is based must be better justified and made explicit. The manuscript will be revised and improved in order to answer to the Reviewer's requests. This important issue regroups several points: rainfall fields, urban network of ML, simulation of path integrated attenuation (PIA) "data" on the ML.

- The study has been performed with data sample regrouping 207 actual rainfall fields observed by a weather radar (the radar pixel is $250 \mathrm{~m} \times 250 \mathrm{~m}$ ). These rainfall fields are representative of the types of rain events in the region of Nantes. They have been selected so as to cover the range of rainfall variability observed in this region, including the intermittency of rainfall fields (Emmanuel et al., 2012). According to the reviewer request, the manuscript will be revised in order to clarify this point.

- The urban network of ML is the actual network of the city of Nantes (database CartoRadio - French National Frequency Agency). If its realism cannot be discussed, we fully agree with the Reviewer that it introduces the question of the topology of the network. For instance, it is clear that its topology is very different from the topology of the virtual network used by Cuccoli et al. (2013). A solution would be to extend this feasibility study to different cities, equipped with networks of different topologies, or better analyze the topology of the urban ML networks. Let us notice that, to the best of our knowledge, this influence of the network topology has not yet addressed in the literature. The manuscript will be revised in order to rise this point, and to emphasize that the validity of the obtained results is not general, but limited to the context of this

Printer-friendly version

Discussion paper 
study.

- The numerical simulation of path integrated attenuation (PIA) "data". The different sources of errors of PIA which occur along the ML are presented in a very detailed way by Zinevich et al. (2010), Leijnse et al. (2008), Leijnse et al. (2010) and the manuscript refers to these publications. The global measurement error is the combination of these sources of errors, and thus can be reasonably represented by a Gaussian distribution. This distribution must be characterized by a simple way, then we followed hypothesis of an error standard-deviation proportional to the attenuation. This hypothesis meets a reason of simplicity. It is convenient to cover a wide range of errors and it is often retained in numerical experiments. In addition, a more detailed modeling of the measurement error would have requested a more detailed representation of the various sources of errors, and of their possible correlations, which seems impossible without a careful data analysis. Let us notice that the quantization noise is not assumed proportional to the PIA value. The Reviewer considers that the retained magnitude of the measurement error is questionable. Tests have been performed with larger errors (quantization noise of $1 \mathrm{~dB}$ and coefficient alpha=0.2). The obtained results will be detailed in the revised version. Another important issue is the presence of a spatial organization of the errors. As the measurement errors between two independent ML can reasonably be assumed independent, the possible existence of this spatial structure would be related to the spatial organization of the parameters $A$ and $b$ of the attenuation equation $A=a R^{\wedge} b$ which depend on the link frequency and of the rainfall Drop Size Distribution. We have explored this option, but we haven't found enough information to introduce it in a simple way in the numerical experiment. We have therefore neglected an eventual spatial structure. This assumption will also be better explained in the revised version.

- This discussion might be the opportunity to better define the objectives of a feasibility study based on numerical experiments. In our mind, it doesn't replace an evaluation with real data, and the objective is to prepare this real-world evaluation and to identify

Printer-friendly version

Discussion paper 
the factors important for that purpose. It explains why we have adopted a numerical experiment, calling strong hypothesis but remaining simple and introducing a limited number of parameters. The pertinence of taking into account realistic errors in a numerical experiment is also a point of discussion. Numerical experiments have been performed without introducing errors (Cuccoli et al., 2013). In that condition, the feasibility would be equivalent to the evaluation of our capability to reconstruct rainfall fields in an ideal context, and would contribute to assess the potential of a given ML network for mapping rainfall. We think that it could be interesting to introduce these points in the discussion on numerical experiments in a revised version of the manuscript. The specific comments of the Reviewer that are related with this discussion will be taken into account, and the manuscript will be revised according to his/her requests.

4. Proofreading. We are surprised by this comment because the manuscript has been proofread by a native US translator, with whom we are used to work.

Specific comments: Thank you for this very thorough analysis of the manuscript. All these comments and suggestions will be taken into account in the revised version of the manuscript. Some of these specific comments would deserve an answer. We hope that these answers have been provided in the previous paragraphs.

\section{References}

Cuccoli, F., et al., Tomography applied to radiobase network for real time estimation of the rainfal rate fields, Atmos. Res. (2011), doi:10.1016/j.atmosres.2011.06.02.

Leijnse, H., Uijlenhoet, R. \& Stricker, J. N. M., 2008. Microwave link rainfall estimation: Effects of link length and frequency, temporal sampling, power resolution, and wet antenna attenuation. Adv. \Water Resour., 31(11), pp. 1481-1493.

Leijnse, H., Uijlenhoet, R. \& Berne, A., 2010. Errors and uncertainties in microwave link rainfall estimation explored using drop size measurements and high-resolution radar data. Journal of Hydrometeorology, 11(6), pp. 1330-1344.

Printer-friendly version

Discussion paper . 
Menke, W., 1989. Geophysical Data Analysis: Discrete Inverse Theory. s.I.:Elsevier Science.

Messer, H., Zinevitch, A., and P. Alpert, 2012, Environmental Sensor Networks Using Existing Wireless Communication Systems for Rainfall and Wind Velocity Measurements, IEEE Instrumentation \& Measurement Magazine, April 2012, 32-38.

Tarantola, A. \& Valette, B., 1982a. Generalized nonlinear inverse problems solved using the least squares criterion. Rev. Geophys. Space Phys, 20(2), pp. 219-232.

Vignal, B., Andrieu, H., Delrieu, G. and Creutin, J. D.: Identification of RainRate Profiles from Radar Returns at Attenuating Wavelengths Using an Inverse Method: A Feasibility Study, ., 42(7), 1014-1030, J. Appl. Meteorol., doi:10.1175/1520- 0450(2003).

Zinevich, A., Alpert, P. \& Messer, H., 2008. Estimation of rainfall fields using commercial microwave communication networks of variable density. Advances in water resources, 31(11), pp. 1470-1480.

Zinevich, A., Messer, H. \& Alpert, P., 2010. Prediction of rainfall intensity measurement errors using commercial microwave communication links. Atmospheric Measurement Techniques, 3(5), pp. 1385-1402.

\section{Response to Reviewer \#2}

1. We might have missed some references. More details would have been useful. An additional bibliographic scan can be made.

2. The terms used to formulate the identification algorithm are the following. We want to describe a physical system by a set of parameters (scalar or vector) which are therefore the unknowns of the problem. The a priori information groups the information what we may know on these parameters, and which serves to initialize the algorithm. This identification is performed thanks to data (observations) and a model (m) which relates the data and the parameters. For that application of the inverse algorithm, the parameters to identify are regrouped in a vector containing the rainfall rates for all the 
pixels of the study domain. The data regroup the measurement of the path integrated attenuation due to rainfall along the ML. The model is the expression which relates the specific attenuation to rainfall rate $\left(A=a R^{\wedge} b\right)$; this model introduces two constants a and $b$, which take different values according to the frequency of the link. The Reviewer is right, this rule is not followed in the manuscript while line $8 p 4,(a, b)$ are called parameters instead of constant. We will correct it in the revised version to prevent any confusion.

3. The grid nesting is a simple way to refine the resolution when the quantity of information is better while keeping a global view of the rainfall field. It is made possible by the a priori information. From purely numerical point of view, it should be possible to iterate again to reduce the pixel size if information is available at a smaller scale. The model is nearly linear and should not be an obstacle to this refinement. From a more physical point of view, the limit is certainly in the presence of local data.

4. The difficulty was to find an indicator to quantify the level of information brought by a link to a given pixel. For each pixel, the weight is the sum of the proportion of the links which intersect this pixel. The total of all the weights is equal to the number of links. We agree that the denomination "pixel density" is not adapted and could be replaced by "link density" by pixel. The manuscript will be revised according to this comment.

5. This comment indicates that the presentation of the numerical experiment is not clear enough and deserves to be better explained in the revised version of the manuscript.

6. The standard-deviation of the measurement error is assumed proportional to the measured PIA. It means that the link frequency effect is indirectly taken into account, and that the statement on page 10 is not correct. This sentence will be removed.

7. The definition of the decorrelation distance might lead to an ambiguity due to Equation 10 which is erroneous. The variables involved in the inverse algorithm are the residuals $[R-R 0]$ between the a priori rainfall and true rainfall (that we would like to

Printer-friendly version

Discussion paper

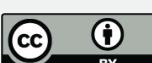


identify). These residuals are characterized by their 'a priori' statistical characteristics, namely a standard-deviation and a covariance matrix, which introduces the decorrelation distance. It means that the decorrelation distance is not the decorrelation distance of the rain field, but the decorrelation distance of the field of residuals between the a priori rain field and the actual rain field. These two decorrelation distances are similar when the initialization is done with a constant value, otherwise they are different. Equation 10 will be corrected. More generally, the influence of the rainfall variability would deserve to be better explained, as suggested by the Reviewer.

Interactive comment on Hydrol. Earth Syst. Sci. Discuss., https://doi.org/10.5194/hess-2016540, 2016. 